\title{
Serum sodium variability and acute kidney injury: a retrospective observational cohort study on a hospitalized population
}

\author{
Gianmarco Lombardi ${ }^{1} \cdot$ Pietro Manuel Ferraro ${ }^{2,3}$ (D) Alessandro Naticchia ${ }^{2} \cdot$ Giovanni Gambaro $^{1}$
}

Received: 11 March 2020 / Accepted: 24 July 2020 / Published online: 9 August 2020

(c) The Author(s) 2020

\begin{abstract}
Aim of our study was to analyze the association between serum sodium (Na) variability and acute kidney injury (AKI) development. We performed a retrospective observational cohort study on the inpatient population admitted to Fondazione Policlinico Universitario A. Gemelli IRCCS between January 1, 2010 and December 31, 2014 with inclusion of adult patients with $\geq 2 \mathrm{Na}$ and $\geq 2$ serum creatinine measurements. We included only patients with $\geq 2 \mathrm{Na}$ measurements before AKI development. The outcome of interest was AKI. The exposures of interest were hyponatremia, hypernatremia and Na fluctuations before AKI development. Na variability was evaluated using the coefficient of variation (CV). Multivariable Cox proportional hazards and logistic regression models were fitted to obtain hazard ratios (HRs), odds ratios (ORs) and 95\% confidence intervals (CIs) for the association between the exposures of interest and AKI. Overall, 56,961 patients met our inclusion criteria. During 1541 person-years of follow-up AKI occurred in 1450 patients. In multivariable hazard models, patients with pre-existent dysnatremia and those who developed dysnatremia had a higher risk of AKI compared with patients with normonatremia. Logistic models suggested a higher risk for AKI in the 3rd (OR 1.41, 95\% CI 1.18, 1.70, $p<0.001)$ and 4th (OR 1.53, 95\% CI 1.24, 1.91, $p<0.001)$ highest quartiles of $\mathrm{Na} \mathrm{CV}$ with a significant linear trend across quartiles $(p$ trend $<0.001)$. This association was also independent from $\mathrm{Na}$ highest and lowest peak value. Dysnatremia is a common condition and is positive associated with AKI development. Furthermore, high Na variability might be considered an independent early indicator for kidney injury development.
\end{abstract}

Keywords Acute kidney injury $\cdot$ Electrolyte disorders $\cdot$ Hyponatremia $\cdot$ Hypernatremia $\cdot$ Epidemiological study

\section{Introduction}

Electrolyte disturbances are common disorders in the hospitalized population [1]. Serum sodium ( $\mathrm{Na}$ ) imbalance is frequently observed in the hospital setting [2]. Dysnatremia conditions (including hyponatremia $[\mathrm{Na}<135 \mathrm{mEq} / \mathrm{L}]$ and

Electronic supplementary material The online version of this article (https://doi.org/10.1007/s11739-020-02462-5) contains supplementary material, which is available to authorized users.

Pietro Manuel Ferraro

pietromanuel.ferraro@unicatt.it

1 U.O.C. Nefrologia, Azienda Ospedaliera Universitaria Integrata di Verona, Verona, Italy

2 U.O.C. Nefrologia, Fondazione Policlinico Universitario A. Gemelli IRCCS, Via G. Moscati 31, 00168 Rome, Italy

3 Università Cattolica del Sacro Cuore, Rome, Italy hypernatremia $[\mathrm{Na}>145 \mathrm{mEq} / \mathrm{L}]$ are reported in approximately $30-40 \%$ of all hospital admissions [3].

Medical and scientific interest on these conditions is justified by the significant burden of Na disorders on the patient's prognosis [4]. Both hyponatremia and hypernatremia have been widely associated with increased morbidity and mortality. Furthermore, as suggested by recently published studies, even small fluctuations in serum $\mathrm{Na}$ levels have been associated with a significant increase of in-hospital mortality [5-8].

As the main organ involved in water metabolism and homeostasis, the kidney is generally the main culprit for such disorders. Defective urine dilution with disproportionally high water intake causes hyponatremia. On the other way around, disorders involving urine concentration with inadequately low water intake cause hypernatremia $[9,10]$. Therefore, it is not surprising that kidney diseases, especially acute kidney injury (AKI), characterized by an abrupt 
reduction in renal function, are commonly associated with these pathological conditions [11-15].

On the other hand, as sparsely reported in medical literature, an inverse relationship between dysnatremia and AKI emerges, where $\mathrm{Na}$ imbalance precedes and predicts kidney damage. A plausible cross-talk on a biological and pathogenetic ground might justify such relationship [16-18], but it still remains poorly investigated $[14,19]$.

The aim of our study is to analyze the association between dysnatremia, in the whole range of its manifestations (hyponatremia, hypernatremia and mild Na fluctuations in the normonatremic range), and AKI development using a large retrospective cohort of hospitalized patients.

\section{Methods}

\section{Setting and study population}

We performed a retrospective observational study on the hospitalized population admitted to Fondazione Policlinico Universitario A. Gemelli IRCCS, a tertiary level hospital serving more than 1 million people in Rome, between January 1, 2010 and December 31, 2014. We included only adult patients (aged 18 years or older) with at least two serum $\mathrm{Na}$ (with consensual serum glucose) and at least two serum creatinine measurements during hospital stay. For analysis and data calculation we included only patients with at least two Na measurements before AKI development. Patients with end-stage kidney disease (ESKD) were excluded. Study patients were included at the time of their first hospital admission. If a patient was hospitalized multiple times during the study period, we considered only the first one.

\section{Data collection}

All data were extracted from the hospital electronic database. We exported the following demographic, clinical and laboratory data: age, sex, serum $\mathrm{Na}$, glucose, creatinine, primary and secondary ICD-9-CM (International Classification of Disease, 9th Revision, Clinical Modification) diagnosis codes at hospital discharge.

\section{Definitions}

Acute kidney injury (AKI) was defined according to creatinine kinetics criteria [20].

In-hospital AKI was defined as AKI developed after $\geq 48 \mathrm{~h}$ from hospital admission.

Patients were grouped according to all $\mathrm{Na}$ values recorded during hospital stay and preceding AKI development in the following dysnatremic groups: hyponatremia $(\mathrm{Na}$ value $<135 \mathrm{mmol} / \mathrm{L})$, hypernatremia $(\mathrm{Na}$ value $>145 \mathrm{mmol} / \mathrm{L}$ ), normonatremia (lowest/highest $\mathrm{Na}$ values $\geq 135 \mathrm{mmol} / \mathrm{L}$ and $\leq 145 \mathrm{mmol} / \mathrm{L}$ ). In patients with mixed dysnatremia, only the first $\mathrm{Na}$ disorder (what happened first), hypo or hypernatremia, was considered.

All Na levels were corrected for the dilutional effect associated with hyperglycemia using a validated method [21].

$\mathrm{Na}$ variability (or fluctuations) was evaluated using the coefficient of variation $(\mathrm{CV})$, defined as the ratio between the standard deviation and the mean of all $\mathrm{Na}$ values preceding AKI development.

Comorbid conditions (cardiovascular diseases, malignancies, gastrointestinal diseases, genitourinary disorders, endocrine/metabolic disorders, infectious and respiratory diseases) were identified using ICD-9-CM codes. The Charlson/Deyo comorbidity index score [22] was calculated for each hospital admission using primary and secondary diagnosis ICD-9-CM codes at hospital discharge.

ESKD was identified using administrative data (ICD-9 CM codes using chronic ESKD criteria: procedure codes for arteriovenous fistula creation or revision $(39.27,39.42$, 39.43, and 39.93); history of ESKD requiring either kidney transplant (identified through discharge diagnosis ICD-9 V42.0) or maintenance dialysis (ICD-9 V45.1, V45.11, V45.12, V56, V56.0, V56.8). Dialysis criteria were: any of the following procedure codes: 39.95 (hemodialysis), V45.1 (renal dialysis status), V56.0 (extracorporeal dialysis), or V56.1 (fitting and adjustment of extracorporeal dialysis catheter); the initiation of dialysis in a patient with no known history of prior dialysis (ICD-9p 39.95, 54.98)) or laboratory data (baseline estimated glomerular filtration rate $[\mathrm{eGFR}]<15 \mathrm{~mL} / \mathrm{min} / 1.73 \mathrm{~m}^{2}$ ). The baseline GFR was estimated for each hospital admission with the CKD-EPI formula [23] using the first creatinine value read at hospital admission.

\section{Outcomes and covariates}

The outcome of interest was in-hospital AKI development.

The exposures of interest were the dysnatremia groups and $\mathrm{Na}$ fluctuations (expressed as quartiles of $\mathrm{CV}$ and analyzed as categorical and numeric variable).

The covariates that were used for risk adjustment in multivariable regression analyses were: age, sex, Charlson/Deyo score, comorbidities, Na value at hospital admission, eGFR at hospital admission. Survival time was defined as the time from in-hospital admission to primary outcome, loss to follow-up or censoring, whichever occurred first.

\section{Statistical analysis}

Categorical variables were expressed as numbers and percentages. Continuous variables were expressed as means with standard deviations (SDs) (normal distribution) or 
medians with interquartile ranges (IQRs) (skewed distribution). Normality of distributions was evaluated by visual inspection of histogram and Q-Q plot.

To explore the association between dysnatremia and AKI, we used a cause-specific regression hazard model. To confirm our results, as sensitivity analysis, we performed subdistribution hazard models considering in-hospital death as competitive risk. Cumulative acute kidney outcome was obtained using the cumulative incidence function for competitive risk. Time at risk started when Na disorder (hypoor hypernatremia) was first observed, in normonatremic patients at first Na measurement. All alive patients were censored at the time of hospital discharge. Unadjusted and multivariable adjusted hazard ratios (HRs) with $95 \%$ confidence interval (95\% CI) were reported for all survival analyses.

A logistic regression model, unadjusted and adjusted for all covariates, was fitted to obtain the odds ratios (ORs) and $95 \% \mathrm{CI}$ of the association between the quartiles of $\mathrm{Na}$ $\mathrm{CV}$ and in-hospital AKI. Three models were built: Model 1 represents unadjusted ORs; Model 2 was adjusted for age, sex, comorbidities, Na value at hospital admission, baseline eGFR; Model 3 was adjusted for Na lowest and highest peak value in addition to factors included in Model 2. The first $\mathrm{CV}$ quartile was used as reference. A $p$ value for trend was calculated by treating quartiles as continuous variables in each model.

To evaluate the modification effects of subgroups on the relationship between dysnatremia and AKI development, a preplanned subgroup analysis was performed. The study population was stratified in the following subgroups: age ( $<60$ or $\geq 60$ years old), sex (men or women), comorbidities (cardiovascular diseases, malignancies, gastrointestinal diseases, genitourinary disorders, endocrine/metabolic disorders, infectious and respiratory diseases), baseline eGFR ( $<60$ or $\geq 60 \mathrm{~mL} / \mathrm{min} / 1.73 \mathrm{~m}^{2}$ ).

For analysis and data calculation, we used $\mathrm{R}$ statistics (version 3.4.4, R Foundation for Statistical Computing Platform). A $p$ value $<0.05$ was considered as statistically significant.

\section{Ethical}

The ethics committee of Fondazione Policlinico Universitario A. Gemelli IRCCS approved the research protocol (Prot. number 34327/18 ID 2210).

\section{Results}

A total of 56,961 patients met our inclusion criteria. Table 1 presents the baseline characteristics of the study population. In total, 22,068 (38.7\%) had cardiovascular diseases, 18,070 (31.7\%) had malignancies, $9120(16.0 \%)$ had gastrointestinal diseases, $6328(11.1 \%)$ had genitourinary disorders, 10,070 (17.7\%) had endocrine/metabolic disorders, 3049 (5.4\%) infectious diseases, 7625 (13.4\%) respiratory diseases, with

Table 1 Baseline characteristics of the study population

\begin{tabular}{|c|c|c|c|c|c|}
\hline & $\begin{array}{l}\text { All } \\
n=56,961\end{array}$ & $\begin{array}{l}\text { Normonatremia } \\
n=44,178\end{array}$ & $\begin{array}{l}\text { Hyponatremia } \\
n=8803\end{array}$ & $\begin{array}{l}\text { Hypernatremia } \\
n=3980\end{array}$ & $p$ value \\
\hline Age, years, mean (SD) & $60.9(18.0)$ & $59.3(18.0)$ & $65.7(17.4)$ & $67.8(15.6)$ & $<0.001$ \\
\hline Males, $n(\%)$ & $26,632(46.8)$ & $20,368(46.1)$ & $4411(50.1)$ & $1853(46.6)$ & $<0.001$ \\
\hline Charlson/Deyo score, $n(\%)$ & & & & & $<0.001$ \\
\hline 0 & $43,957(77.2)$ & $35,156(79.6)$ & $6138(69.7)$ & $2663(66.9)$ & \\
\hline 1 & $9082(15.9)$ & $6477(14.7)$ & $1692(19.2)$ & 913 (22.9) & \\
\hline 2 & $2169(3.8)$ & $1456(3.3)$ & $470(5.3)$ & $243(6.1)$ & \\
\hline$>2$ & $1753(3.1)$ & $1089(2.5)$ & $503(5.7)$ & $161(4.0)$ & \\
\hline \multicolumn{6}{|l|}{ Comorbidities, $n(\%)$} \\
\hline Cardiovascular & $22,068(38.7)$ & $16,263(36.8)$ & $3795(43.1)$ & $2010(50.5)$ & $<0.001$ \\
\hline Malignancies & $18,070(31.7)$ & $13,672(30.9)$ & $3166(36.0)$ & $1232(31.0)$ & $<0.001$ \\
\hline Gastrointestinal & $9120(16.0)$ & $6757(15.3)$ & $1874(21.3)$ & $489(12.3)$ & $<0.001$ \\
\hline Genitourinary & $6328(11.1)$ & $4593(10.4)$ & $1175(13.3)$ & $560(14.1)$ & $<0.001$ \\
\hline Endocrine/metabolic & $10,070(17.7)$ & $7520(17.0)$ & 1709 (19.4) & $841(21.1)$ & $<0.001$ \\
\hline Infectious & $3049(5.4)$ & $1740(3.9)$ & $1008(11.5)$ & $301(7.6)$ & $<0.001$ \\
\hline Respiratory & $7625(13.4)$ & $4871(11.0)$ & $1781(20.2)$ & $973(24.4)$ & $<0.001$ \\
\hline $\begin{array}{l}\text { eGFR, mean (SD), mL/ } \\
\mathrm{min} / 1.73 \mathrm{~m}^{2 *}\end{array}$ & $79.6(25.9)$ & $81.4(25.2)$ & $75.0(28.1)$ & $70.7(25.5)$ & $<0.001$ \\
\hline $\mathrm{Na}$, median (IQR), mEq/L* & $140.0(138.0,142.0)$ & $140.0(139.0,142.0)$ & $135.0(132.0,139.0)$ & $144.0(141.0,146.0)$ & $<0.001$ \\
\hline $\mathrm{Na} C \mathrm{~V}$, median (IQR) & $1.3(0.7,1.9)$ & $1.0(0.5,1.5)$ & $2.3(1.7,3.0)$ & $2.1(1.5,2.9)$ & $<0.001$ \\
\hline
\end{tabular}

*Value at hospital admission 
a mean eGFR at hospital admission of 79.6 (SD 25.9) $\mathrm{mL} /$ $\mathrm{min} / 1.73 \mathrm{~m}^{2}$ and a median Na value at hospital admission of $140 \mathrm{mEq} / \mathrm{L}$ (IQR 138.0-142.0, range lowest-highest 101.0-175.0 mEq/L) (Table 1).

In the study population, 44,178 stayed in a normonatremic condition while in $12,783(22.4 \%)$ a dysnatremic status occurred (hyponatremia in 8803 [15.5\%], hypernatremia in 3980 [7.0\%]). Dysnatremic patients aged older (59.3 years [SD 18.0], 65.7 years [SD 17.4], 67.8 years [SD 15.6], in normo-, hypo-, hypernatremics, respectively, $p<0.001)$ with a higher prevalence in males $(46.1 \%, 50.1 \%$, $46.6 \%$, in normo-, hypo- and hypernatremics, respectively, $p<0.001$ ). Hyponatremia, and hypernatremia showed a worse comorbidity index, with a higher prevalence in cardiovascular diseases $(36.8 \%, 43.1 \%, 50.5 \%$ in normo-, hypo- and hypernatremics, respectively, $p<0.001$ ), malignancicies $(30.9 \%, 36.0 \%, 31.0 \%$ in normo-, hypo- and hypernatremics, respectively, $p<0.001$ ), genitourinary disorders $(10.4 \%, 13.3 \%, 14.1 \%$ in normo-, hypo- and hypernatremics, respectively, $p<0.001$ ), endocrine/metabolic disorders $(17.0 \%, 19.4 \%, 21.1 \%$ in normo-, hypo- and hypernatremics, respectively, $p<0.001)$, infectious diseases $(3.9 \%, 11.5 \%$, $7.6 \%$ in normo-, hypo- and hypernatremics, respectively, $p<0.001)$, respiratory diseases $(11.0 \%, 20.2 \%, 24.4 \%$ in normo-, hypo- and hypernatremics, respectively, $p<0.001$ ). Lower eGFR was observed in patients with Na disorders $(81.4 \mathrm{~mL} / \mathrm{min}$ [SD 25.2], $75.0 \mathrm{~mL} / \mathrm{min}$ [SD 28.1], $70.7 \mathrm{~mL} /$ min [SD 25.5] in normo-, hypo- and hypernatremics, respectively, $p<0.001$ ).

Coefficient of $\mathrm{Na}$ variation ( $\mathrm{Na} \mathrm{CV}$ ) was used to describe $\mathrm{Na}$ fluctuation in the study cohort. Patients with higher $\mathrm{Na}$ variability showed worse comorbidty index and higher prevalence in comorbidities. Older age and lower baseline eGFR was also observed in subjects with higher $\mathrm{Na}$ fluctuations (Table 2).

\section{Sodium and AKI}

\section{Dysnatremia and AKI}

During 1541 years of follow-up, the outcome occurred in 1450 (2.5\%, incidence rate 940.9 per 1000 person-year).

A dysnatremic condition was associated with increased risk of AKI development (Table 3). Patients with dysnatremia or patients who developed dysnatremia had a higher risk of AKI occurrence with an $\mathrm{HR}$, in multivariable adjusted model, of 1.87 (95\% CI 1.61, 2.16, $p<0.001$ ), HR 1.67 (95\% CI 1.41, 1.98, $p<0.001$ ), in hyponatremia and hypernatremia, respectively.

\section{Sodium variability and AKI}

In Table 4, we investigated the association between Na variability and AKI. Unadjusted regression models suggested a

Table 2 Baseline characteristics of the study population stratified by quartile of Na coefficient of variation (CV)

\begin{tabular}{|c|c|c|c|c|c|}
\hline & $\begin{array}{l}1 \mathrm{st} \\
\mathrm{CV} \leq 0.71 \\
n=14,580\end{array}$ & $\begin{array}{l}2 \mathrm{nd} \\
0.71<\mathrm{CV} \leq 1.25 \\
n=14,037\end{array}$ & $\begin{array}{l}\text { 3rd } \\
1.25<\mathrm{CV} \leq 1.94 \\
n=14,206\end{array}$ & $\begin{array}{l}4 \text { th } \\
\text { CV }>1.94 \\
n=14,138\end{array}$ & $p$ value \\
\hline Age, years, mean (SD) & $57.8(18.3)$ & $60.5(17.9)$ & $62.1(17.3)$ & $63.2(18.0)$ & $<0.001$ \\
\hline Men, $n(\%)$ & $6587(45.2)$ & $6806(48.5)$ & $6925(48.7)$ & $6314(44.7)$ & $<0.001$ \\
\hline Charlson/Deyo score, $n(\%)$ & & & & & $<0.001$ \\
\hline 0 & $11,771(80.7)$ & $10,869(77.4)$ & $10,754(75.7)$ & $10,563(74.7)$ & \\
\hline 1 & $2063(14.1)$ & $2232(15.9)$ & $2375(16.7)$ & $2412(17.1)$ & \\
\hline 2 & $411(2.8)$ & $531(3.8)$ & $585(4.1)$ & $642(4.5)$ & \\
\hline$>2$ & $335(2.3)$ & $405(2.9)$ & $492(3.5)$ & $521(3.7)$ & \\
\hline \multicolumn{6}{|l|}{ Comorbidities, $n(\%)$} \\
\hline Cardiovascular & $5130(35.2)$ & $5424(38.6)$ & 5837 (41.1) & $5677(40.2)$ & $<0.001$ \\
\hline Malignancies & $4151(28.5)$ & $4440(31.6)$ & $4808(33.8)$ & $4671(33.0)$ & $<0.001$ \\
\hline Gastrointestinal & $2307(15.8)$ & $2229(15.9)$ & $2342(16.5)$ & $2242(15.9)$ & 0.363 \\
\hline Genito/urinary & $1408(9.7)$ & $1502(10.7)$ & $1617(11.4)$ & $1801(12.7)$ & $<0.001$ \\
\hline Endocrine/metabolic & $2466(16.9)$ & $2441(17.4)$ & 2655 (18.7) & $2508(17.7)$ & 0.001 \\
\hline Infectious & $496(3.4)$ & $632(4.5)$ & $820(5.8)$ & $1101(7.8)$ & $<0.001$ \\
\hline Respiratory & $1277(8.8)$ & $1654(11.8)$ & $2122(14.9)$ & $2572(18.2)$ & $<0.001$ \\
\hline eGFR, mean (SD), $\mathrm{mL} / \mathrm{min} / 1.73 \mathrm{~m}^{2} *$ & $83.0(25.0)$ & $80.1(25.5)$ & $78.1(25.8)$ & $77.3(27.1)$ & $<0.001$ \\
\hline $\mathrm{Na}$, median (IQR), mEq/L* & $140.0(139.0,142.0)$ & $140.0(138.0,142.0)$ & $140.0(138.0,142.0)$ & $139.0(136.0,142.0)$ & $<0.001$ \\
\hline $\mathrm{NaCV}$, median (IQR) & $0.5(0.0,0.5)$ & $1.0(1.0,1.1)$ & $1.5(1.5,1.7)$ & $2.5(2.1,3.1)$ & $<0.001$ \\
\hline
\end{tabular}

*Value at hospital admission 
Table 3 Association of dysnatremia with AKI development

\begin{tabular}{lllll}
\hline & Overall & Normonatremia & Hyponatremia & Hypernatremia \\
\hline No. of patients & 56,961 & 44,178 & 8803 & 3980 \\
Person-years & 1541 & 1167 & 269 & 106 \\
Time to AKI, median (IQR), days & - & - & $8.0(10.0)$ & $7.0(8.0)$ \\
Kidney outcome & & & $381(4.3)$ & $205(5.2)$ \\
AKI (N, \%) & $1450(2.5)$ & $864(2.0)$ & 1416.4 & 1934.0 \\
AKI per 1000 person-year & 940.9 & 740.4 & & \\
Cause-specific hazard model & & & $1.98(1.76,2.24)$ & $2.69(2.31,3.13)$ \\
HR (95\% CI & - & 1.00 (Reference) & $p<0.001$ & $p<0.001$ \\
& - & 1.00 (Reference) & $1.87(1.61,2.16)$ & $1.67(1.41,1.98)$ \\
HR (95\% CI)* & & & $p<0.001$ & $p<0.001$ \\
\hline
\end{tabular}

*Adjusted for: age, sex, comorbidities, Na value at hospital admission, eGFR at baseline

Table 4 Association between Na variability (CV) and AKI development

\begin{tabular}{|c|c|c|c|c|c|c|c|}
\hline & \multirow{2}{*}{$\begin{array}{l}\text { No. of events } \\
(\%)\end{array}$} & \multicolumn{2}{|l|}{ Model 1} & \multicolumn{2}{|l|}{ Model 2} & \multicolumn{2}{|l|}{ Model 3} \\
\hline & & OR $(95 \%$ CI $)$ & $\begin{array}{l}p \text { value } \\
\text { for trend }\end{array}$ & OR $(95 \% \mathrm{CI})$ & $\begin{array}{l}p \text { value for } \\
\text { trend }\end{array}$ & OR $(95 \%$ CI $)$ & $p$ value for trend \\
\hline $\begin{array}{l}\text { Q1 } \\
\text { CV } \leq 0.71\end{array}$ & 209 (1.4) & $\begin{array}{l}1.00 \text { (Refer- } \\
\text { ence) }\end{array}$ & $<0.001$ & 1.00 (Reference) & $<0.001$ & 1.00 (Reference) & $<0.001$ \\
\hline $\begin{array}{l}\text { Q2 } \\
0.71<\mathrm{CV} \leq 1.25\end{array}$ & $289(2.1)$ & $\begin{array}{l}1.45(121,1.73) \\
p<0.001\end{array}$ & & $\begin{array}{l}1.25(1.04,1.50) \\
p=0.017\end{array}$ & & $\begin{array}{l}1.16(0.97,1.40) \\
p=0.115\end{array}$ & \\
\hline $\begin{array}{l}\mathrm{Q} 3 \\
1.25<\mathrm{CV} \leq 1.94\end{array}$ & 418 (2.9) & $\begin{array}{c}2.08(1.77, \\
2.47) \\
p<0.001\end{array}$ & & $\begin{array}{l}1.66(1.40,1.97) \\
p<0.001\end{array}$ & & $\begin{array}{l}1.41(1.18,1.70) \\
p<0.001\end{array}$ & \\
\hline $\begin{array}{l}\text { Q4 } \\
\text { CV > } 1.94\end{array}$ & $534(3.8)$ & $\begin{array}{c}2.70(2.30 \\
3.18) \\
p<0.001\end{array}$ & & $\begin{array}{l}2.12(1.79,2.51) \\
p<0.001\end{array}$ & & $\begin{array}{l}1.53(1.24,1.91) \\
p<0.001\end{array}$ & \\
\hline
\end{tabular}

Model 1: Unadjusted model

Model 2: Multivariable adjusted logistic regression. Adjusted for age, sex, comorbidities, Na value at hospital admission, eGFR baseline

Model 3: Multivariable adjusted logistic regression. Adjusted for age, sex, comorbidities, Na value at hospital admission, eGFR baseline, Na lowest and highest peak value

higher risk for AKI development in the 2nd (OR 1.45, 95\% CI 1.21, 1.73, $p<0.001$ ), 3rd (OR 2.08, 95\% CI 1.77, 2.47, $p<0.001)$ and 4th (OR 2.70, 95\% CI 2.30, 3.18, $p<0.001)$ quartile of $\mathrm{Na} \mathrm{CV}$. This association was also observed in the multivariable adjusted model (Model 2) with an OR of 1.25 (95\% CI 1.04, 1.50, $p=0.017)$, an OR of 1.66 (95\% CI $1.40,1.97, p<0.001)$ and an OR of 2.12 (95\% CI 1.79, 2.51, $p<0.001$ ) in the $2 \mathrm{nd}, 3 \mathrm{rd}$ and in the 4 th quartile of $\mathrm{Na} \mathrm{CV}$ respectively with a significant linear trend across all quartiles ( $p$ for trend $<0.001$ ). Of note, even after adjustment for Na lowest and highest peak value (Model 3) the significant and independent relationship was confirmed.

\section{Subgroup analysis}

We evaluated the modification effects of subgroups on the relationship between dysnatremia and AKI development performing subgroup analysis (Table 5). A significant interaction was observed in dysnatremic patients older than 60 years, with cardiovascular and endocrine/metabolic disorders; there was also an effect modification only in hyponatremic patients male, with gastrointestinal diseases, genito/urinary disorders and $\mathrm{eGFR}<60 \mathrm{~mL} / \mathrm{min}$.

\section{Sensitivity analysis}

As sensitivity analysis a subdistribution hazard model between AKI development and dysnatremic groups was built (Supplemental Table 1) using death as competitive risk. The relationship between the outcome of interest and the exposures was confirmed, with a significant and independent increase risk of AKI development in dysnatremic groups (HR 1.84, 95\% CI $1.58,2.16, p<0.001$, HR $1.62,95 \%$ CI $1.35,1.95, p<0.001$, respectively in hyponatremia and hypernatremia condition). 
Table 5 Subgroup associations of dysnatremia with AKI development

\begin{tabular}{|c|c|c|c|c|}
\hline & \multicolumn{2}{|c|}{ AKI (hyponatremia sample) } & \multicolumn{2}{|c|}{ AKI (hypernatremia sample) } \\
\hline & HR $(95 \%$ CI $)$ & $p$ for interaction & HR $(95 \% \mathrm{CI})$ & $p$ for interaction \\
\hline Age $\geq 60$ & $4.32(3.26,5.71)$ & $<0.001$ & $4.26(2.80,6.48)$ & 0.003 \\
\hline Age $<60$ & $1.53(1.34,1.76)$ & & $2.14(1.81,2.52)$ & \\
\hline Male & $2.58(2.12,3.14)$ & $<0.001$ & $3.23(2.53,4.13)$ & 0.075 \\
\hline Female & $1.65(1.41,1.92)$ & & $2.43(2.00,2.95)$ & \\
\hline Cardiovascular & $3.35(2.73,4.10)$ & $<0.001$ & $4.39(3.36,5.73)$ & $<0.001$ \\
\hline No cardiovascular & $1.42(1.22,1.66)$ & & $1.85(1.53,2.23)$ & \\
\hline Malignancies & $1.85(1.60,2.15)$ & 0.069 & $2.73(2.30,3.26)$ & 0.652 \\
\hline No malignancies & $2.36(1.91,2.92)$ & & $2.52(1.84,3.45)$ & \\
\hline Gastrointestinal & $1.85(1.61,2.11)$ & 0.005 & $2.68(2.28,3.15)$ & 0.960 \\
\hline No gastrointestinal & $2.93(2.19,3.91)$ & & $2.71(1.97,4.40)$ & \\
\hline Genito/urinary & $2.04(1.78,2.34)$ & 0.031 & $2.75(2.32,3.27)$ & 0.089 \\
\hline No genito/urinary & $1.47(1.13,1.92)$ & & $2.00(1.44,2.77)$ & \\
\hline Endocrine/metabolic & $2.25(1.96,2.59)$ & $<0.001$ & $3.10(2.61,3.70)$ & 0.001 \\
\hline No endocrine/metabolic & $1.34(1.05,1.71)$ & & $1.71(1.25,2.34)$ & \\
\hline Infectious & $2.08(1.83,2.37)$ & 0.085 & $2.59(2.20,3.05)$ & 0.314 \\
\hline No infectious & $1.46(1.00,2.14)$ & & $3.29(2.12,5.11)$ & \\
\hline Respiratory & $2.21(1.93,2.54)$ & $<0.001$ & $2.71(2.24,3.28)$ & 0.100 \\
\hline No respiratory & $1.23(0.96,1.59)$ & & $2.06(1.58,2.69)$ & \\
\hline $\mathrm{eGFR} \geq 60$ & $1.37(1.17,1.61)$ & $<0.001$ & $1.95(1.61,2.36)$ & 0.123 \\
\hline $\mathrm{eGFR}<60$ & $2.28(1.89,2.73)$ & & $2.51(1.94,3.24)$ & \\
\hline
\end{tabular}

\section{Discussion}

Our study demonstrates a strong and independent association between $\mathrm{Na}$ disturbances and $\mathrm{Na}$ variability with the risk of AKI development. Dysnatremia, hyponatremia or hypernatremia are significantly associated with acute kidney injury and higher Na variability (CV) predicts AKI development during hospital stay.

AKI is a complex clinical syndrome characterized by a sudden reduction in renal function and defined as an increase (absolute or relative) of creatinine levels or a reduction in urinary output [24]. The high burden on in-hospital patient prognosis $[25,26]$ justifies the medical and scientific interest in this clinical syndrome. Since the kidney is the main organ engaged in fluid and electrolyte homeostasis, it is not surprising that renal dysfunctions are frequently associated with water imbalance and so alterations in serum electrolyte levels. In particular, hypo and hypernatremia conditions, often observed in the hospitalized population [1], have been related to kidney injury [11-15].

A growing body of recent evidences highlight the relationship between disorders of metabolism and electrolyte homeostasis and strong outcomes [8, 27, 28]. Several studies have described the association between $\mathrm{Na}$ balance and kidney function [11-13] however, a few of them have considered dysnatremia as predictor of AKI [14, 19] and none has investigated $\mathrm{Na}$ variability as a marker in kidney injury development.
During 1541 person-years of follow-up, AKI was observed in $1450(2.5 \%)$ patients (incidence rate 940.9 per 1000 person-year); 588 (40.4\%) of them showed a dysnatremia condition (at hospital admission or during hospital stay) before AKI development. As clearly demonstrated by our results, Na disturbances were strongly and independently associated with in-hospital AKI. Of note, higher Na fluctuations were linearly related to an increased risk of AKI independently of $\mathrm{Na}$ peak value.

Many pathological conditions may share dysnatremia and AKI such as volume overload or depletion, heart and liver diseases, infectious diseases and partly explain our findings. Under specific conditions, such as cardiovascular diseases, cardio-renal syndrome, gastrointestinal diseases or malignancies, hyponatremia has been associated with AKI development [11-13]. According to our findings, older patients, with cardiovascular diseases and endocrine/metabolic disorders with dysnatremia were especially associated with increased risk of AKI.

However, an independent relationship between Na disturbances and Na variability emerges from our study. Recently Lee et al., focusing only on an hyponatremic hospitalized population, described an independent correlation between dysnatremia and AKI development, where pre-existing hyponatremia increased the risk of AKI by $30 \%$ [19].

Using our retrospective cohort, we decided to go further these findings. Not only pre-existing hyponatremia condition was associated with kidney injury, but also hypernatremia 
demonstrated as a potential independent predictor of AKI. Interestingly, higher $\mathrm{Na}$ variability was often observed before kidney damage. From this point of view, since serum creatinine is such a poor sensitive marker of kidney damage, higher $\mathrm{Na}$ fluctuations may anticipate worsening of renal function.

An appropriate question should be whether dysnatremia is a simple bystander or contributing factor of AKI. Dysnatremia and kidney injury may be two different manifestations of a common underlying disease or reflect the severity of the illness and comorbidities of the patient. Volume depletion that generally accompanies hypernatremia is frequently recognized as a common cause of AKI development. On the other hand, Na dilution (or true Na depletion due to diuretics) can be observed in systemic disorders (i.e., heart, liver failure) causing AKI, characterized by increased extracellular volume.

However, according to our results, although we could not exclude residual confounding due to the retrospective nature of the study, a direct pathogenic relationship between $\mathrm{Na}$ imbalance and kidney injury might be hypothesized. Concerning this, it is interesting to note the median time between dysnatremia and the onset of AKI (7-8 days), as reported on Table 3. An interesting finding in our study, Na variability and kidney injury were associated independently from $\mathrm{Na}$ peak value (highest or lowest), in accordance with what has been shown by other Authors [8, 27], suggesting a potentially more damaging role of $\mathrm{Na}$ rapid variations rather than $\mathrm{Na}$ absolute value. Evidence suggests that osmotic stress can cause cellular damage, even if we do not know the exact mechanism at kidney level. The direct effect of serum sodium concentration and variation on extracellular tonicity is well known. Serum sodium fluctuations induce water shift in and out of the cells threatening their survival [29-31]. A wide variety of experimental models have demonstrated that osmo-stress can evoke multiple apoptotic pathways, inhibit anti-apoptotic gene expression, induce multiple cytokines and reactive oxygen species generation [32-34]. Even if we do not know the exact mechanism of osmotic damage at kidney level, it would be consistent with the reported evidence; however, it has yet to be elucidated in detail. Further studies, with a prospective design, are required to carefully explore and confirm such causal association.

Several study limitations have to be reported such as a retrospective and monocentric design, the use of ICD-9-CM codes to identify comorbid conditions, unavailability of information on chronic or acute therapy administered during hospital stay. However, the use of Charlson/Deyo comorbidity index score to account for patient comorbidity (a validated index of diseases severity based on ICD-9-CM codes), a wide general in-hospital cohort and a creatinine-based definition of in-hospital AKI give strength to our results.
To our knowledge, this is the first study that has widely analyzed the association between Na disorders and kidney injury demonstrating that: (i) dysnatremia is a common condition that involves AKI patients; and (ii) high Na variability might be considered a good biological marker that anticipates kidney injury development.

Acknowledgements Open access funding provided by Universit $\tilde{A}$ Cattolica del Sacro Cuore within the CRUI-CARE Agreement. G.L., P.M.F. and G.G. contributed to the research idea and study design. G.L. contributed to data acquisition. G.G., P.M.F. and G.L. contributed to data analysis/interpretation. P.M.F. and G.L. contributed to statistical analysis. G.L. drafted the paper. G.G., P.M.F. and A.N. contributed to supervision. Each author contributed important intellectual content during manuscript drafting for the overall work.

\section{Compliance with ethical standards}

Conflict of interest The authors declare that they have no conflict of interest.

Human and animal rights statement The study protocol and the waiver of consent were approved by the ethics committee of Fondazione Policlinico Universitario A. Gemelli IRCCS (Approval No. 34327/18 ID 2210). The study was conducted in accordance with Declaration of Helsinki.

Informed consent This study waived the requirement for written informed consent due to the retrospective nature of this study.

Open Access This article is licensed under a Creative Commons Attribution 4.0 International License, which permits use, sharing, adaptation, distribution and reproduction in any medium or format, as long as you give appropriate credit to the original author(s) and the source, provide a link to the Creative Commons licence, and indicate if changes were made. The images or other third party material in this article are included in the article's Creative Commons licence, unless indicated otherwise in a credit line to the material. If material is not included in the article's Creative Commons licence and your intended use is not permitted by statutory regulation or exceeds the permitted use, you will need to obtain permission directly from the copyright holder. To view a copy of this licence, visit http://creativecommons.org/licenses/by/4.0/.

\section{References}

1. Funk G-C, Lindner G, Druml W et al (2010) Incidence and prognosis of dysnatremias present on ICU admission. Intensive Care Med 36:304-311. https://doi.org/10.1007/s00134-009-1692-0

2. Spasovski G, Vanholder R, Allolio B et al (2014) Clinical practice guideline on diagnosis and treatment of hyponatraemia. Nephrol Dial Transplant 29(Suppl 2):i1-i39. https://doi.org/10.1093/ndt/ gfu040

3. Upadhyay A, Jaber BL, Madias NE (2006) Incidence and prevalence of hyponatremia. Am J Med 119:S30-35. https://doi. org/10.1016/j.amjmed.2006.05.005

4. Wald R, Jaber BL, Price LL et al (2010) Impact of hospitalassociated hyponatremia on selected outcomes. Arch Intern Med 170:294-302. https://doi.org/10.1001/archinternmed.2009.513 
5. Marshall DC, Salciccioli JD, Goodson RJ et al (2017) The association between sodium fluctuations and mortality in surgical patients requiring intensive care. J Crit Care 40:63-68. https:// doi.org/10.1016/j.jcrc.2017.02.012

6. Sakr Y, Rother S, Ferreira AMP et al (2013) Fluctuations in serum sodium level are associated with an increased risk of death in surgical ICU patients. Crit Care Med 41:133-142. https://doi. org/10.1097/CCM.0b013e318265f576

7. Topjian AA, Stuart A, Pabalan AA et al (2014) Greater fluctuations in serum sodium levels are associated with increased mortality in children with externalized ventriculostomy drains in a PICU. Pediatr Crit Care Med 15:846-855. https://doi.org/10.1097/ PCC.0000000000000223

8. Lombardi G, Ferraro PM, Calvaruso L et al (2019) sodium fluctuations and mortality in a general hospitalized population. Kidney Blood Press Res 44:604-614. https://doi.org/10.1159/000500916

9. Guyton AC, Hall JE (2006) Textbook of medical physiology, 11th edn. Elsevier Saunders, Philadelphia

10. Skorecki K, Chertow GM, Marsden PA (2016) Brenner \& Rector's The Kidney, 10th edn. Elsevier, Philadelphia

11. Aronson D, Darawsha W, Promyslovsky M et al (2014) Hyponatraemia predicts the acute (type 1) cardio-renal syndrome. Eur J Heart Fail 16:49-55. https://doi.org/10.1093/eurjhf/hft123

12. Lameire N, Van Biesen W, Vanholder R (2010) Electrolyte disturbances and acute kidney injury in patients with cancer. Semin Nephrol 30:534-547. https://doi.org/10.1016/j.semnephrol .2010.09.002

13. Hackworth WA, Heuman DM, Sanyal AJ et al (2009) Effect of hyponatraemia on outcomes following orthotopic liver transplantation. Liver Int 29:1071-1077. https://doi.org/10.111 1/j.1478-3231.2009.01982.x

14. Adams D, de Jonge R, van der Cammen $T$ et al (2011) Acute kidney injury in patients presenting with hyponatremia. J Nephrol 24:749-755. https://doi.org/10.5301/JN.2011.6410

15. Libório AB, Silva GB, Silva CGCH et al (2012) Hyponatremia, acute kidney injury, and mortality in HIV-related toxoplasmic encephalitis. Braz J Infect Dis 16:558-563. https://doi. org/10.1016/j.bjid.2012.08.015

16. Dunn FL, Brennan TJ, Nelson AE, Robertson GL (1973) The role of blood osmolality and volume in regulating vasopressin secretion in the rat. J Clin Investig 52:3212-3219. https://doi. org/10.1172/JCI107521

17. Fitzsimons JT (1998) Angiotensin, thirst, and sodium appetite. Physiol Rev 78:583-686. https://doi.org/10.1152/physr ev.1998.78.3.583

18. Lee Y-J, Song I-K, Jang K-J et al (2007) Increased AQP2 targeting in primary cultured IMCD cells in response to angiotensin II through AT1 receptor. Am J Physiol Renal Physiol 292:F340-350. https://doi.org/10.1152/ajprenal.00090.2006

19. Lee SW, Baek SH, Ahn SY et al (2016) The effects of pre-existing hyponatremia and subsequent-developing acute kidney injury on in-hospital mortality: a retrospective cohort study. PLoS ONE 11:e0162990. https://doi.org/10.1371/journal.pone.0162990

20. Waikar SS, Bonventre JV (2009) Creatinine kinetics and the definition of acute kidney injury. J Am Soc Nephrol 20:672-679. https ://doi.org/10.1681/ASN.2008070669
21. Hillier TA, Abbott RD, Barrett EJ (1999) Hyponatremia: evaluating the correction factor for hyperglycemia. Am J Med 106:399-403

22. Deyo RA, Cherkin DC, Ciol MA (1992) Adapting a clinical comorbidity index for use with ICD-9-CM administrative databases. J Clin Epidemiol 45:613-619

23. Silveiro SP, Araújo GN, Ferreira MN et al (2011) Chronic kidney disease epidemiology collaboration (CKD-EPI) equation pronouncedly underestimates glomerular filtration rate in type 2 diabetes. Diabetes Care 34:2353-2355. https://doi.org/10.2337/ dc11-1282

24. Bellomo R, Kellum JA, Ronco C (2012) Acute kidney injury. Lancet 380:756-766. https://doi.org/10.1016/S0140-6736(11)61454 $-2$

25. Medve L, Antek C, Paloczi B et al (2011) Epidemiology of acute kidney injury in Hungarian intensive care units: a multicenter, prospective, observational study. BMC Nephrol 12:43. https://doi. org/10.1186/1471-2369-12-43

26. Hoste EAJ, Clermont G, Kersten A et al (2006) RIFLE criteria for acute kidney injury are associated with hospital mortality in critically ill patients: a cohort analysis. Crit Care 10:R73. https:// doi.org/10.1186/cc4915

27. Thongprayoon C, Cheungpasitporn W, Yap JQ, Qian Q (2019) Increased mortality risk associated with serum sodium variations and borderline hypo- and hypernatremia in hospitalized adults. Nephrol Dial Transplant. https://doi.org/10.1093/ndt/gfz098

28. Lombardi G, Ferraro PM, Bargagli M et al (2019) Hyperchloremia and acute kidney injury: a retrospective observational cohort study on a general mixed medical-surgical not ICU-hospitalized population. Intern Emerg Med. https://doi.org/10.1007/s11739-01902165-6

29. Burg MB, Ferraris JD, Dmitrieva NI (2007) Cellular response to hyperosmotic stresses. Physiol Rev 87:1441-1474. https://doi. org/10.1152/physrev.00056.2006

30. Strange K (2004) Cellular volume homeostasis. Adv Physiol Educ 28:155-159. https://doi.org/10.1152/advan.00034.2004

31. Watanabe K, Umeda T, Niwa K et al (2018) A PP6-ASK3 module coordinates the bidirectional cell volume regulation under osmotic stress. Cell Rep 22:2809-2817. https://doi.org/10.1016/j.celre p.2018.02.045

32. Shapiro L, Dinarello CA (1995) Osmotic regulation of cytokine synthesis in vitro. Proc Natl Acad Sci USA 92:12230-12234. https ://doi.org/10.1073/pnas.92.26.12230

33. López-Rodríguez C, Aramburu J, Jin L et al (2001) Bridging the NFAT and NF-kappaB families: NFAT5 dimerization regulates cytokine gene transcription in response to osmotic stress. Immunity 15:47-58. https://doi.org/10.1016/s1074-7613(01)00165-0

34. Zhang W-C, Zheng X-J, Du L-J et al (2015) High salt primes a specific activation state of macrophages, M(Na). Cell Res 25:893910. https://doi.org/10.1038/cr.2015.87

Publisher's Note Springer Nature remains neutral with regard to jurisdictional claims in published maps and institutional affiliations. 